\title{
SINTOMAS VOCAIS E FATORES RELATIVOS AO ESTILO DE VIDA EM PROFESSORES
}

\author{
Vocal symptoms and factors related to teachers' lifestyle
}

\author{
Carollina Caporossi ${ }^{(1)}$, Léslie Piccolotto Ferreira ${ }^{(2)}$
}

\begin{abstract}
RESUMO
Objetivo: identificar os hábitos vocais autorreferidos por professores, e associar os mesmos a presença de rouquidão, cansaço ao falar, garganta seca e alteração de voz, também autorreferidos. Métodos: 88 professores de duas escolas de ensino fundamental e médio da rede pública do Município de Sorocaba responderam o questionário CPV-P composto de 84 questões, e parte dessas foi analisada (dados pessoais; situação funcional; aspectos vocais e hábitos). Na análise estatística, as variáveis dependentes foram a autorreferencia a alteração de voz, rouquidão, cansaço ao falar, e garganta seca, e as independentes sexo, idade, situação funcional e hábitos. A análise estatística, realizada no programa STATA 8.0 por meio do teste de associação (qui-quadrado) considerou nível de $p \leq 0,05$. Resultados: $64,77 \%$ dos sujeitos fizeram autorreferencia a alteração vocal, $54,55 \%$ a cansaço ao falar, $53,41 \%$ a garganta seca, $44,32 \%$ a rouquidão. Dentre os hábitos inadequados, 86,52 autorreferiram falar muito; 59,55\%, falar em lugar aberto; e 50,56\%, gritar. Em contrapartida, $67,42 \%$ poupam a voz, e $75,28 \%$ bebem água durante o uso da voz. Foi possível observar a associação entre a autorreferência a presença de alteração vocal e ser mulher (valor de $p=0,018$ ) e falar muito (valor de $p=0,033$ ); entre cansaço ao falar e ser mulher (valor de $p=0,017$ ) e mais idoso (valor de $p=0,012$ ); entre garganta seca e ser mais idoso(valor de $p=0,033$ ). Conclusão: os hábitos referidos em maior número foram os de falar muito, em lugar aberto e gritar. As variáveis ser mulher, mais idoso e falar muito estiveram associadas a parte dos sintomas analisados.
\end{abstract}

DESCRITORES: Docentes; Distúrbios da Voz; Fatores de Risco

\section{INTRODUÇÃO}

A profissão do docente é uma das que apresenta maior incidência de alterações vocais, em sua maioria em função das condições de trabalho, em grande parte, inadequadas, como falar durante horas, em intensidade elevada em consequência do

(1) Aluna do Curso de Graduação em Fonoaudiologia da Pontifícia Universidade Católica de São Paulo, PUC-SP, São Paulo, SP, Brasil.

(2) Fonoaudióloga; Professora Titular do Departamento de Fundamentos da Fonoaudiologia da Pontifícia Universidade Católica de São Paulo, PUC-SP, São Paulo, SP, Brasil; Coordenadora e Docente do Curso de Especialização em Fonoaudiologia - Voz da Coordenadoria Geral de Especialização, Aperfeiçoamento e Extensão da Pontifícia Universidade Católica de São Paulo, COGEAE/PUC-SP, São Paulo, SP, Brasil; Mestre em Linguística Aplicada pela Pontifícia Universidade Católica de São Paulo; Doutora em Distúrbios da Comunicação Humana pela Universidade Federal de São Paulo.

Conflito de interesses: inexistente ruído ambiental. Pesquisas brasileiras realizadas com professores, que na área da Fonoaudiologia são em número significativo ${ }^{1,2}$, evidenciam que as manifestações desse distúrbio podem ser: esforço à emissão, dificuldade de manter a voz, variação na frequência fundamental, habitual ou na intensidade, rouquidão, falta de volume e projeção, perda de eficiência vocal e pouca resistência ao falar. Internacionalmente as pesquisas também confirmam esses dados ${ }^{3}$.

Entre os professores, os sintomas mais comuns são: rouquidão, pigarro/tosse, dor de garganta/ ardor, fadiga vocal, garganta seca, perda de voz ou afonia e variação na emissão vocal. Esses sintomas são mencionados em diferentes pesquisas, com porcentagens que variam de acordo com o grupo de professores pesquisados ${ }^{4,5}$, entre os sintomas mais citados estão a rouquidão, o cansaço vocal e a garganta seca ${ }^{4,6}$.Dentre os fatores de risco para o aparecimento desses sintomas, além dos ambientais e de organização do trabalho ${ }^{7}$, o estilo de vida, 
representado por hábitos, é mencionado e trabaIhado nas ações fonoaudiológicas de promoção de saúde ou prevenção de distúrbios de voz. Destaque especial é dado ao tabagismo, ao etilismo e a falta de hidratação, além de hábitos inadequados de uso vocal, como gritar, falar alto ou muito ${ }^{8}$. Especificamente entre os professores, no entanto, principalmente entre aqueles que atuam junto ao ensino fundamental, parece haver pouca ingestão de álcool e tabagismo, fato que leva a hipótese de que outros fatores possam explicar a presença de sintomas.

Os fonoaudiólogos estimulam a manutenção de hábitos como ingerir boa quantidade de água, ter uma alimentação saudável e regular, evitar alimentos condimentados e choques térmicos por meio de alimentos ou líquidos e não falar em quadros gripais ou alérgicos. Da mesma forma, competir com ruído, e o hábito de tossir ou pigarrear aumentam o atrito das pregas vocais, o que acaba sendo um fator de risco para a ocorrência do distúrbio de $\mathrm{voz}^{9}$.

Dessa forma, um comportamento vocal adequado seria aquele que contaria com a hidratação do corpo, além de atenção quanto a avaliar a interferência na produção da voz, de falar em ambientes secos e empoeirados, gritar sem suporte respiratório, falar com golpes de glote, tossir ou pigarrear excessivamente, falar em lugares ruidosos ou abertos, usar tons graves ou agudos demais, falar excessivamente durante quadros gripais ou crises alérgicas, praticar exercícios físicos falando, fumar ou falar muito em ambientes de fumantes, ingerir bebida alcoólica em excesso, falar demasiadamente, rir alto, falar muito após ingerir grandes quantidades de aspirinas, calmantes ou diuréticos, discutir com frequência, entre outros ${ }^{8,9}$.

Um problema sério é a falta de qualquer tipo de preparo ou orientação quanto ao uso da voz no sentido de sensibilizar e conscientizar o professor. Grillo e Penteado (2005) ${ }^{10}$ destacam que ações para minimizar os problemas vocais de professores devem começar no período da formação do professor e se estender por toda sua carreira. O índice de alterações vocais somado ao impacto gerado na vida profissional desses sujeitos faz com que, cada vez mais, os professores sejam afastados de sala de aula. Carneiro (2006) ${ }^{11}$ destaca que $97 \%$ das readaptações e $62 \%$ das licenças no município de São Paulo são de profissionais relacionadas ao ensino.

Reconhecer os fatores que interfiram na produção vocal é de suma importância, uma vez que, quando detectados, é possível planejar ações de prevenção de distúrbios vocais e a promoção da saúde vocal. Nessa direção, a Prefeitura de Sorocaba preocupada em mapear os problemas dos professores, propôs a implantação de um Programa de Saúde Vocal do Professor, com o objetivo de melhorar a qualidade de vida dos professores e, concomitantemente, cuidar do bem estar vocal dos mesmos. Considerando a parceria estabelecida entre o LaborVox (PUC-SP) e a referida Prefeitura o levantamento de dados referentes aos hábitos presentes entre os professores poderá ser um dos passos para subsidiar as ações a serem planejadas para sensibilizar o professor quanto às questões de bem estar vocal e conseqüentemente minimizar distúrbios de voz presentes entre eles. Numa visão multiplicadora, as ações poderão ainda ser ampliadas para também beneficiar o bem estar vocal de alunos e funcionários que convivem no mesmo ambiente.

Dessa forma, o objetivo deste estudo foi identificar os hábitos vocais auto-referidos por professores do ensino fundamental e médio, e associa-los à presença de rouquidão, cansaço ao falar, garganta seca e alteração de voz, também auto-referidos.

\section{MÉTODOS}

Realizou-se um estudo de corte transversal em professores do ensino fundamental e médio da Rede Municipal de Ensino do município de Sorocaba. Duas escolas dessa rede foram selecionadas para participarem da pesquisa. Todos os 103 professores que atuavam na época da coleta de dados foram convidados a participarem.

Foram adotados como critérios de inclusão, fazer parte do corpo docente das escolas pesquisadas; estar em atividade plena e regular; frequentar regularmente as reuniões semanais denominadas Horário de Trabalho Pedagógico Coletivo (HTPC), momento esse em que as instruções sobre a pesquisa seriam dadas; e concordar em participar da pesquisa assinando o termo de consentimento livre e esclarecido. Como critério de exclusão foi considerado apenas a não finalização, por qualquer motivo, dos procedimentos de coleta de dados.

Ao final participaram 88 professores do ensino fundamental, uma vez que 15 professores não se disponibilizaram a responder o instrumento.

Para coleta de dados foi utilizado o questionário denominado CPV-P proposto por Ferreira et al. $(2007)^{12}$, composto por 84 questões, separadas em dimensões, a saber: identificação do questionário; características do professor; características da organização do trabalho; características físicas do local de trabalho; aspectos perceptivos e de uso vocal; aspectos gerais de saúde; antecedentes mórbidos; e fatores relativos ao estilo de vida.

Especificamente para este estudo foram consideradas as seguintes questões: sexo, idade, 
tempo de magistério, número de escolas em que o professor leciona, carga horária semanal, tabagismo, etilismo, número de refeições diárias, horas de sono; gritar, falar muito, falar em lugar aberto, falar durante atividade física, falar carregando peso, poupar a voz, e beber água enquanto fala.

Em sua maioria, as questões foram apresentadas em escala ordinal com a solicitação de marcar a frequência das ocorrências (0. nunca 1 . raramente 2 . às vezes 3 . sempre 4 . não sei).

Antes da aplicação do questionário, os professores foram informados sobre os objetivos da pesquisa, e o caráter voluntário e sigiloso da participação de cada um foi garantido pela assinatura de termo de compromisso.

A aplicação do questionário foi realizada na própria escola onde os professores lecionavam, em horários designados para as reuniões pedagógicas denominadas de Horário de Trabalho Pedagógico Coletivo (HTPC).

O projeto foi aprovado pelo Comitê de Ética em Pesquisa da PUC/SP (processo no 08/700).

Os dados obtidos com os questionários foram tabulados no programa Microsoft Office Excel 2003, e optou-se por digitação dupla para eliminar a ocorrência de erros.

$\mathrm{Na}$ análise estatística, foi realizada a associação entre as variáveis dependentes (auto-referência de alteração de voz e presença dos sintomas de rouquidão, cansaço ao falar, e garganta seca) e as variáveis independentes de sexo, idade (analisadas quanto ao grupo maior e menor que a idade média), tempo de magistério (idem quanto ao tempo médio), número de escolas que leciona, carga horária semanal (idem quanto a media de horas), tabagismo, etilismo, quantidade de refeições diárias, horas de sono (idem quanto a media de horas), hábito de poupar a voz, gritar, falar muito, falar em lugar aberto, falar em atividade física, falar carregando peso e beber água durante o uso da voz. Para tanto, com o auxilio do programa STATA, versão 8.0 (Stata Corp., College Station, Estados Unidos) foi utilizado o teste de Qui-Quadrado e em todas as análises foi considerado nível de significância de 5\%.

\section{RESULTADOS}

O grupo de 88 professores pesquisados foi caracterizado como sendo composto por $70,45 \%$ (62) do sexo feminino e $29,55 \%$ (26) do masculino. A média de idade dos mesmos foi de 42 anos de idade (mínima de 22 anos e máxima de 66 anos). O tempo médio de magistério referido pelos professores foi de 14 anos (tempo mínimo $<1$ ano e máximo de 35 anos), e a média de horas de jornada de trabalho de 42 horas semanais (mais da metade dos entrevistados, ou seja, 52,27\%(46) atuam em carga horária maior que 30 horas semanais).

Dentre os entrevistados, $64,77 \%$ (57) do grupo fez autorreferência à presença de alteração de voz, no presente ou no passado, e dentre os sintomas pesquisados, $54,55 \%(48)$ assinalaram a presença de cansaço ao falar, $53,41 \%$ (47) de garganta seca e $44,32 \%$ (39) de rouquidão.

A Tabela 1 detalha essas ocorrências em correlação as variáveis: sexo, idade, tempo de magistério, número de escolas que leciona e carga horária semanal. Assinala ainda que foi encontrada associação estatística significante entre alteração vocal auto-referida $(p=0,018)$ e cansaço ao falar $(p=0,017)$ e o sexo feminino, e tendência $(p=0,062)$ quanto a presença do sintoma de rouquidão, a favor do mesmo sexo.

Houve, ainda, associação estatisticamente significante entre a autorreferência a cansaço ao falar e garganta seca e o grupo de professores representado pelos mais velhos (respectivamente $p=0,012$ e $p=0,033$ ).

Quanto ao estilo de vida, aqui mapeado pelos hábitos que podem interferir de forma negativa na produção da voz, dentre os entrevistados, 77 $(86,52 \%)$, fizeram autorreferência a falar muito, $59,55 \%$ (53) a falar em lugar aberto, 50,56\% (45) a gritar, $28,41 \%(25)$ a fumar, $28,09 \%$ (25) a falar carregando peso, e $22,73 \%$ (20) a ingerir bebida alcoólica.

Em contrapartida, dentre os hábitos preconizados como positivos para a produção vocal, $75,28 \%$ (67) disseram beber água durante o uso da voz, e $67,42 \%$ (60) poupam a voz.

$\mathrm{Na}$ leitura da Tabela 2 pode-se identificar essas ocorrências e a associação delas com a autorreferência a alteração vocal, rouquidão, cansaço ao falar e garganta seca. Foi encontrada associação estatística significante entre alteração vocal autorreferida e o falar muito $(p=0,033)$.

\section{DISCUSSÃO}

A porcentagem de professores que fizeram referência a manifestar, no presente ou passado, alteração vocal é semelhante a outras pesquisas realizadas com professores ${ }^{6,13,14}$, fato que, mais uma vez, confirma que os professores fazem parte de um grupo de risco para distúrbios vocais. Em especial, na comparação com a pesquisa realizada no município de São Paulo ${ }^{15}$, que contou com 422 professores e utilizou o mesmo instrumento para coleta de dados, pode-se constatar a semelhança frente a $60 \%$ dos entrevistados terem feito referencia no presente ou no passado à alteração vocal. 
Tabela 1 - Distribuição numérica e percentual dos professores quanto ao sexo (M- Masculino; FFeminino), idade (maior e menor à média do grupo), tempo de magistério (maior e menor à média do grupo), número de escolas que leciona (maior e menor à média do grupo), e carga horária semanal (maior e menor à média do grupo), segundo a autorreferência de alteração de voz, rouquidão, cansaço ao falar e garganta seca

\begin{tabular}{|c|c|c|c|c|c|c|c|c|c|c|c|c|c|}
\hline \multirow[b]{3}{*}{ Variável } & \multirow[b]{3}{*}{ Categoria } & \multicolumn{12}{|c|}{ Autorreferencia } \\
\hline & & \multicolumn{3}{|c|}{ Alteração de voz } & \multicolumn{3}{|c|}{ Rouquidão } & \multicolumn{3}{|c|}{ Cansaço ao falar } & \multicolumn{3}{|c|}{ Garganta seca } \\
\hline & & $\begin{array}{c}\text { não } \\
\text { n } \\
(\%)\end{array}$ & $\begin{array}{c}\text { Sim } \\
n \\
(\%)\end{array}$ & $\mathrm{P}$ & $\begin{array}{c}\text { não } \\
n \\
(\%)\end{array}$ & $\begin{array}{c}\operatorname{sim} \\
n \\
(\%)\end{array}$ & $\mathrm{P}$ & $\begin{array}{c}\text { não } \\
n \\
(\%)\end{array}$ & $\begin{array}{c}\text { Sim } \\
n \\
(\%)\end{array}$ & $\mathrm{P}$ & $\begin{array}{c}\text { não } \\
\text { n } \\
(\%)\end{array}$ & $\begin{array}{c}\operatorname{sim} \\
n \\
(\%)\end{array}$ & $\mathrm{P}$ \\
\hline \multirow[t]{2}{*}{ Sexo } & $M$ & $\begin{array}{c}14 \\
(53.85)\end{array}$ & $\begin{array}{c}12 \\
(46.15)\end{array}$ & $0.018^{\star}$ & $\begin{array}{c}17 \\
(68.00)\end{array}$ & $\begin{array}{c}8 \\
(32.00)\end{array}$ & $0.062 \#$ & $\begin{array}{c}15 \\
(62.50)\end{array}$ & $\begin{array}{c}9 \\
(37.50)\end{array}$ & $0.017^{\star}$ & $\begin{array}{c}13 \\
(54.17)\end{array}$ & $\begin{array}{c}11 \\
(45.83)\end{array}$ & 0.176 \\
\hline & $\mathrm{F}$ & $\begin{array}{c}17 \\
(27.42)\end{array}$ & $\begin{array}{c}45 \\
(72.58)\end{array}$ & & $\begin{array}{c}26 \\
(45.61)\end{array}$ & $\begin{array}{c}31 \\
(54.39)\end{array}$ & & $\begin{array}{c}20 \\
(33.90)\end{array}$ & $\begin{array}{c}39 \\
(66.10)\end{array}$ & & $\begin{array}{c}22 \\
(37.93)\end{array}$ & $\begin{array}{c}36 \\
(62.07)\end{array}$ & \\
\hline \multirow[t]{2}{*}{$\begin{array}{l}\text { Idade } \\
\text { (anos) }\end{array}$} & $\geq 42$ & $\begin{array}{c}12 \\
(28.57)\end{array}$ & $\begin{array}{c}30 \\
(71.43)\end{array}$ & 0.390 & $\begin{array}{c}19 \\
(51.35)\end{array}$ & $\begin{array}{c}18 \\
(48.65)\end{array}$ & 0.818 & $\begin{array}{c}11 \\
(28.21)\end{array}$ & $\begin{array}{c}28 \\
(71.79)\end{array}$ & $0.012^{\star}$ & $\begin{array}{c}11 \\
(29.73)\end{array}$ & $\begin{array}{c}26 \\
(70.27)\end{array}$ & $0.033^{*}$ \\
\hline & $<41$ & $\begin{array}{c}15 \\
(37.50)\end{array}$ & $\begin{array}{c}25 \\
(62.50)\end{array}$ & & $\begin{array}{c}19 \\
(48.72)\end{array}$ & $\begin{array}{c}20 \\
(51.28)\end{array}$ & & $\begin{array}{c}22 \\
(56.41)\end{array}$ & $\begin{array}{c}17 \\
(43.59)\end{array}$ & & $\begin{array}{c}21 \\
(53.85)\end{array}$ & $\begin{array}{c}18 \\
(46.15)\end{array}$ & \\
\hline \multirow{2}{*}{$\begin{array}{l}\text { Tempo de } \\
\text { magistério } \\
\text { (anos) }\end{array}$} & $\geq 14$ & $\begin{array}{c}14 \\
(29.17)\end{array}$ & $\begin{array}{c}34 \\
(70.83)\end{array}$ & 0.763 & $\begin{array}{c}19 \\
(42.22)\end{array}$ & $\begin{array}{c}26 \\
(57.78)\end{array}$ & 0.344 & $\begin{array}{c}28 \\
(60.87)\end{array}$ & $\begin{array}{c}18 \\
(39.13)\end{array}$ & 0.874 & $\begin{array}{c}20 \\
(44.44)\end{array}$ & $\begin{array}{c}25 \\
(55.56)\end{array}$ & 0.802 \\
\hline & $<13$ & $\begin{array}{c}17 \\
(43.59) \\
\end{array}$ & $\begin{array}{c}22 \\
(56.41) \\
\end{array}$ & & $\begin{array}{c}19 \\
(52.78) \\
\end{array}$ & $\begin{array}{c}17 \\
(47.22) \\
\end{array}$ & & $\begin{array}{c}19 \\
(52.78) \\
\end{array}$ & $\begin{array}{c}17 \\
(47.22 \\
\end{array}$ & & $\begin{array}{c}15 \\
(41.67) \\
\end{array}$ & $\begin{array}{c}21 \\
(58.33) \\
\end{array}$ & \\
\hline \multirow{2}{*}{$\begin{array}{l}\text { Numero } \\
\text { de escolas } \\
\text { que } \\
\text { leciona }\end{array}$} & $\geq 2$ & $\begin{array}{c}20 \\
(36.36)\end{array}$ & $\begin{array}{c}35 \\
(63.64)\end{array}$ & 0.773 & $\begin{array}{c}24 \\
(48.00)\end{array}$ & $\begin{array}{c}26 \\
(52.00)\end{array}$ & 0.314 & $\begin{array}{c}20 \\
(38.46)\end{array}$ & $\begin{array}{c}32 \\
(61.54)\end{array}$ & 0.376 & $\begin{array}{c}22 \\
(44.00)\end{array}$ & $\begin{array}{c}28 \\
(56.00)\end{array}$ & 0.763 \\
\hline & 1 & $\begin{array}{c}11 \\
(33.33) \\
\end{array}$ & $\begin{array}{c}22 \\
(66.67) \\
\end{array}$ & & $\begin{array}{c}19 \\
(59.38) \\
\end{array}$ & $\begin{array}{c}13 \\
(40.63) \\
\end{array}$ & & $\begin{array}{c}15 \\
(48.39) \\
\end{array}$ & $\begin{array}{c}16 \\
(51.61) \\
\end{array}$ & & $\begin{array}{c}13 \\
(40.63) \\
\end{array}$ & $\begin{array}{c}19 \\
(59.38) \\
\end{array}$ & \\
\hline \multirow{2}{*}{$\begin{array}{l}\text { Carga } \\
\text { horária } \\
\text { semanal } \\
\text { (horas) }\end{array}$} & $\geq 40$ & $\begin{array}{c}10 \\
(37.04)\end{array}$ & $\begin{array}{c}17 \\
(62.96)\end{array}$ & 0.777 & $\begin{array}{c}13 \\
(48.15)\end{array}$ & $\begin{array}{c}14 \\
(51.85)\end{array}$ & 0.473 & $\begin{array}{c}12 \\
(44.44)\end{array}$ & $\begin{array}{c}15 \\
(55.56)\end{array}$ & 0.874 & $\begin{array}{c}13 \\
(48.15)\end{array}$ & $\begin{array}{c}14 \\
(51.85)\end{array}$ & 0.571 \\
\hline & $<39$ & $\begin{array}{c}20 \\
(33.90)\end{array}$ & $\begin{array}{c}39 \\
(66.10)\end{array}$ & & $\begin{array}{c}30 \\
(56.60)\end{array}$ & $\begin{array}{c}23 \\
(43.40)\end{array}$ & & $\begin{array}{c}23 \\
(42.59)\end{array}$ & $\begin{array}{c}31 \\
(57.41)\end{array}$ & & $\begin{array}{c}22 \\
(41.51)\end{array}$ & $\begin{array}{c}31 \\
(58.49)\end{array}$ & \\
\hline
\end{tabular}

Quanto aos sintomas pesquisados, nessa mesma pesquisa, a ocorrência de garganta seca $(57 \%)$ e cansaço ao falar (50\%) também são semelhantes, divergindo apenas quanto à autorreferência ao sintoma de rouquidão (53\%).

Mais uma vez ainda, nesta pesquisa, assim como em outras realizadas com professores, o sexo feminino prevaleceu. Porém, mesmo assim, foi possível propor a associação das demais variáveis a sexo. A maior ocorrência de autorreferência a alteração vocal assim como do sintoma de cansaço ao falar foi suficiente para registrar a diferença significante frente ao sexo feminino, fato que corrobora com os dados da literatura que dizem haver maior prevalência de alteração vocal no sexo feminino ${ }^{6,14-16}$.

Ortiz et al (2004) ${ }^{17}$ apontam em seus estudos, a ocorrência de mudanças na configuração glótica das mulheres durante a fonação prolongada com loudness elevado, possivelmente por diferenças constitucionais e anatômicas. Ainda, os autores justificam o maior número de mulheres devido a área pedagógica ser tradicionalmente do domínio feminino.

É sabido que o envelhecimento é responsável por mudanças no trato vocal e, conseqüentemente na voz, em função de uma série de alterações anatômicas e estruturais que ocorrem com o passar dos anos, como por exemplo, o rebaixamento da laringe e arqueamento das pregas vocais. $\mathrm{Na}$ presente pesquisa, a prevalência de sujeitos com a queixa de cansaço ao falar foi estatisticamente significante em sujeitos com idade maior que 42 anos, ou seja, acima da média de idade dos sujeitos pesquisados. Soares et al $(2007)^{18}$ correlacionam a queixa de cansaço ao falar à idade, pela presença de uma fenda glótica, com o avançar da idade, responsável por mudanças na capacidade respiratória que evidenciam, assim, o sintoma. Os mesmos autores ressaltam que o avanço da idade acarreta num enrijecimento do tórax e enfraquecimento dos músculos respiratórios, o que acaba por favorecer ainda mais a redução da capacidade respiratória. Também, devido ao arqueamento das pregas vocais, decorrente do envelhecimento, é necessário maior esforço para haver coaptação glótica, o que justificaria a queixa de cansaço ao falar. Além desses fatores biológicos, é importante lembrar que a intensa carga horária registrada pelos professores também pode justificar a presença dos sintomas. 
Tabela 2 - Distribuição numérica e percentual dos professores quanto ao tabagismo, etilismo, número de refeições diárias, horas de sono, poupar a voz, gritar, falar muito, falar em lugar aberto e falar durante atividade física, segundo a autorreferência de alteração de voz, rouquidão, cansaço ao falar e garganta seca

\begin{tabular}{|c|c|c|c|c|c|c|c|c|c|c|c|c|c|}
\hline \multirow[b]{3}{*}{ Variável } & \multirow[b]{3}{*}{ Categoria } & \multicolumn{12}{|c|}{ Autorreferencia } \\
\hline & & \multicolumn{3}{|c|}{ Alteração de voz } & \multicolumn{3}{|c|}{ Rouquidão } & \multicolumn{3}{|c|}{ Cansaço ao falar } & \multicolumn{3}{|c|}{ Garganta seca } \\
\hline & & $\begin{array}{c}\text { não } \\
\text { n } \\
(\%)\end{array}$ & $\begin{array}{c}\text { sim } \\
n \\
(\%)\end{array}$ & $\mathbf{P}$ & $\begin{array}{c}\text { não } \\
\text { n } \\
(\%)\end{array}$ & $\begin{array}{c}\text { sim } \\
n \\
(\%)\end{array}$ & $\mathbf{p}$ & $\begin{array}{c}\text { não } \\
\text { n } \\
(\%)\end{array}$ & $\begin{array}{c}\text { Sim } \\
n \\
(\%)\end{array}$ & $\mathbf{p}$ & $\begin{array}{c}\text { não } \\
\text { n } \\
(\%)\end{array}$ & $\begin{array}{c}\text { sim } \\
n \\
(\%)\end{array}$ & $\mathbf{p}$ \\
\hline \multirow[t]{2}{*}{ Fuma } & Não & $\begin{array}{c}24 \\
(77.42)\end{array}$ & $\begin{array}{c}39 \\
(68.42)\end{array}$ & 0.371 & $\begin{array}{c}28 \\
(44.44)\end{array}$ & $\begin{array}{c}30 \\
(47.62)\end{array}$ & 0.399 & $\begin{array}{c}26 \\
(41.27)\end{array}$ & $\begin{array}{c}33 \\
(52.38)\end{array}$ & 0.783 & $\begin{array}{c}27 \\
(42.86)\end{array}$ & $\begin{array}{c}31 \\
(49.21)\end{array}$ & 0.434 \\
\hline & Sim & $\begin{array}{c}7 \\
(22.58)\end{array}$ & $\begin{array}{c}18 \\
(31.58)\end{array}$ & & $\begin{array}{c}15 \\
(60.00)\end{array}$ & $\begin{array}{c}9 \\
(36.00)\end{array}$ & & $\begin{array}{c}9 \\
(36.00)\end{array}$ & $\begin{array}{c}15 \\
(60.00)\end{array}$ & & $\begin{array}{c}8 \\
(32.00)\end{array}$ & $\begin{array}{c}16 \\
(64.00)\end{array}$ & \\
\hline \multirow{2}{*}{$\begin{array}{l}\text { Ingere } \\
\text { bebida } \\
\text { alcoólica }\end{array}$} & Não & $\begin{array}{c}22 \\
(32.35)\end{array}$ & $\begin{array}{c}46 \\
(67.65)\end{array}$ & 0.298 & $\begin{array}{c}34 \\
(50.00)\end{array}$ & $\begin{array}{c}28 \\
(41.18)\end{array}$ & 0.285 & $\begin{array}{c}27 \\
(39.71)\end{array}$ & $\begin{array}{c}36 \\
(52.94)\end{array}$ & 0.447 & $\begin{array}{c}28 \\
41.18\end{array}$ & $\begin{array}{c}34 \\
(50.00)\end{array}$ & 0.277 \\
\hline & Sim & $\begin{array}{c}9 \\
(45.00)\end{array}$ & $\begin{array}{c}11 \\
(55.00)\end{array}$ & & $\begin{array}{c}9 \\
(45.00)\end{array}$ & $\begin{array}{c}11 \\
(55.00)\end{array}$ & & $\begin{array}{c}8 \\
(40.00)\end{array}$ & $\begin{array}{c}12 \\
(60.00)\end{array}$ & & $\begin{array}{c}7 \\
35.00\end{array}$ & $\begin{array}{c}13 \\
(65.00)\end{array}$ & \\
\hline \multirow[t]{2}{*}{$\begin{array}{l}\text { Quantidade } \\
\text { de refeições }\end{array}$} & $\geq 3$ & $\begin{array}{c}14 \\
(42.42\end{array}$ & $\begin{array}{c}32 \\
(57.58)\end{array}$ & 0.405 & $\begin{array}{c}17 \\
(53.13)\end{array}$ & $\begin{array}{c}15 \\
(46.88)\end{array}$ & 0.788 & $\begin{array}{c}11 \\
(33.33)\end{array}$ & $\begin{array}{c}22 \\
(66.67)\end{array}$ & 0.205 & $\begin{array}{c}12 \\
38.71\end{array}$ & $\begin{array}{c}19 \\
(61.29)\end{array}$ & 0.759 \\
\hline & $<2$ & $\begin{array}{c}16 \\
(33.33\end{array}$ & $\begin{array}{c}32 \\
(66.67)\end{array}$ & & $\begin{array}{c}22 \\
(50.00)\end{array}$ & $\begin{array}{c}22 \\
(50.00)\end{array}$ & & $\begin{array}{c}21 \\
(47.73)\end{array}$ & $\begin{array}{c}23 \\
(52.27)\end{array}$ & & $\begin{array}{c}19 \\
42.22\end{array}$ & $\begin{array}{c}26 \\
(57.78)\end{array}$ & \\
\hline \multirow[t]{2}{*}{$\begin{array}{l}\text { Horas de } \\
\text { sono }\end{array}$} & $\geq 6,8$ & $\begin{array}{c}18 \\
(38.30\end{array}$ & $\begin{array}{c}29 \\
(61.70)\end{array}$ & 0.578 & $\begin{array}{c}25 \\
(56.82)\end{array}$ & $\begin{array}{c}19 \\
(43.18)\end{array}$ & 0.466 & $\begin{array}{c}21 \\
(50.00)\end{array}$ & $\begin{array}{c}21 \\
(50.00)\end{array}$ & 0.114 & $\begin{array}{c}20 \\
47.62\end{array}$ & $\begin{array}{c}22 \\
(52.38)\end{array}$ & 0.438 \\
\hline & $<6,7$ & $\begin{array}{c}12 \\
(32.43\end{array}$ & $\begin{array}{c}25 \\
(67.57)\end{array}$ & & $\begin{array}{c}17 \\
(48.57)\end{array}$ & $\begin{array}{c}18 \\
(51.43)\end{array}$ & & $\begin{array}{c}12 \\
(32.43)\end{array}$ & $\begin{array}{c}25 \\
(67.57)\end{array}$ & & $\begin{array}{c}14 \\
38.89\end{array}$ & $\begin{array}{c}22 \\
(61.11)\end{array}$ & \\
\hline \multirow[t]{2}{*}{ Poupa a voz } & Não & $\begin{array}{c}7 \\
(33,33)\end{array}$ & $\begin{array}{c}14 \\
(66.67)\end{array}$ & 0.553 & $\begin{array}{c}11 \\
(52.38)\end{array}$ & $\begin{array}{c}8 \\
(38.10)\end{array}$ & 0.226 & $\begin{array}{c}11 \\
(52.38)\end{array}$ & $\begin{array}{c}9 \\
(42.86)\end{array}$ & 0.597 & $\begin{array}{c}9 \\
(42.86)\end{array}$ & $\begin{array}{c}12 \\
(57.14)\end{array}$ & 0.168 \\
\hline & Sim & $\begin{array}{c}20 \\
(33.90) \\
\end{array}$ & $\begin{array}{c}39 \\
(66.10) \\
\end{array}$ & & $\begin{array}{c}28 \\
(47.46) \\
\end{array}$ & $\begin{array}{c}29 \\
(49.15) \\
\end{array}$ & & $\begin{array}{c}21 \\
(35.59) \\
\end{array}$ & $\begin{array}{c}35 \\
(59.32) \\
\end{array}$ & & $\begin{array}{c}24 \\
(40.68) \\
\end{array}$ & $\begin{array}{c}31 \\
(52.54) \\
\end{array}$ & \\
\hline \multirow[t]{2}{*}{ Gritar } & Não & $\begin{array}{c}14 \\
(34.15)\end{array}$ & $\begin{array}{c}27 \\
(65.85)\end{array}$ & 0.975 & $\begin{array}{c}22 \\
(53.66)\end{array}$ & $\begin{array}{c}18 \\
(43.90)\end{array}$ & 0.483 & $\begin{array}{c}18 \\
(43.90)\end{array}$ & $\begin{array}{c}21 \\
(51.22)\end{array}$ & 0.930 & $\begin{array}{c}16 \\
(39.02)\end{array}$ & $\begin{array}{c}22 \\
(53.66)\end{array}$ & 0.984 \\
\hline & Sim & $\begin{array}{c}16 \\
(36.36) \\
\end{array}$ & $\begin{array}{c}28 \\
(63.64) \\
\end{array}$ & & $\begin{array}{c}19 \\
(43.18) \\
\end{array}$ & $\begin{array}{c}20 \\
(45.45) \\
\end{array}$ & & $\begin{array}{c}16 \\
(36.36) \\
\end{array}$ & $\begin{array}{c}25 \\
(56.82) \\
\end{array}$ & & $\begin{array}{c}18 \\
(40.91) \\
\end{array}$ & $\begin{array}{c}23 \\
(52.27) \\
\end{array}$ & \\
\hline \multirow[t]{2}{*}{ Falar muito } & Não & $\begin{array}{c}7 \\
(70.00)\end{array}$ & $\begin{array}{c}3 \\
(30.00)\end{array}$ & $0.033^{*}$ & $\begin{array}{c}4 \\
(40.00)\end{array}$ & $\begin{array}{c}6 \\
(60.00)\end{array}$ & 0.776 & $\begin{array}{c}3 \\
(30.00)\end{array}$ & $\begin{array}{c}6 \\
(60.00)\end{array}$ & 0.932 & $\begin{array}{c}3 \\
(30.00)\end{array}$ & $\begin{array}{c}5 \\
(50.00)\end{array}$ & 0.509 \\
\hline & Sim & $\begin{array}{c}24 \\
(31.58) \\
\end{array}$ & $\begin{array}{c}52 \\
(68.42) \\
\end{array}$ & & $\begin{array}{c}38 \\
(50.00) \\
\end{array}$ & $\begin{array}{c}32 \\
(42.11)\end{array}$ & & $\begin{array}{c}31 \\
(40.79)\end{array}$ & $\begin{array}{c}41 \\
(53.95) \\
\end{array}$ & & $\begin{array}{c}31 \\
(40.79) \\
\end{array}$ & $\begin{array}{c}41 \\
(53.95) \\
\end{array}$ & \\
\hline \multirow[t]{2}{*}{$\begin{array}{l}\text { Falar em } \\
\text { lugar aberto }\end{array}$} & Não & $\begin{array}{c}13 \\
(38.24)\end{array}$ & $\begin{array}{c}21 \\
(61.76)\end{array}$ & 0.540 & $\begin{array}{c}20 \\
(58.82)\end{array}$ & $\begin{array}{c}11 \\
(32.35)\end{array}$ & 0.499 & $\begin{array}{c}12 \\
(35.29)\end{array}$ & $\begin{array}{c}19 \\
(55.88)\end{array}$ & 0.854 & $\begin{array}{c}14 \\
(41.18)\end{array}$ & $\begin{array}{c}16 \\
(47.06)\end{array}$ & 0.634 \\
\hline & Sim & $\begin{array}{c}18 \\
(34.62)\end{array}$ & $\begin{array}{c}34 \\
(65.38)\end{array}$ & & $\begin{array}{c}22 \\
(42.31)\end{array}$ & $\begin{array}{c}27 \\
(51.92)\end{array}$ & & $\begin{array}{c}22 \\
(42.31)\end{array}$ & $\begin{array}{c}28 \\
(53.85)\end{array}$ & & $\begin{array}{c}20 \\
(38.46)\end{array}$ & $\begin{array}{c}30 \\
(57.69)\end{array}$ & \\
\hline \multirow{2}{*}{$\begin{array}{l}\text { Falar em } \\
\text { atividade } \\
\text { física }\end{array}$} & Não & $\begin{array}{c}24 \\
(39.34)\end{array}$ & $\begin{array}{c}37 \\
(60.66)\end{array}$ & 0.291 & $\begin{array}{c}34 \\
(55.74)\end{array}$ & $\begin{array}{c}23 \\
(37.70)\end{array}$ & 0.377 & $\begin{array}{c}25 \\
(40.98)\end{array}$ & $\begin{array}{c}32 \\
(52.46)\end{array}$ & 0.960 & $\begin{array}{c}24 \\
(39.34)\end{array}$ & $\begin{array}{c}32 \\
(52.46)\end{array}$ & 0.940 \\
\hline & Sim & $\begin{array}{c}7 \\
(29.17) \\
\end{array}$ & $\begin{array}{c}17 \\
(70.83) \\
\end{array}$ & & $\begin{array}{c}8 \\
(33.33) \\
\end{array}$ & $\begin{array}{c}14 \\
(58.33)\end{array}$ & & $\begin{array}{c}9 \\
(37.50)\end{array}$ & $\begin{array}{c}14 \\
(58.33) \\
\end{array}$ & & $\begin{array}{c}10 \\
(41.67)\end{array}$ & $\begin{array}{c}13 \\
(54.17) \\
\end{array}$ & \\
\hline \multirow{2}{*}{$\begin{array}{l}\text { Falar } \\
\text { carregando } \\
\text { peso }\end{array}$} & Não & $\begin{array}{c}26 \\
(37.68)\end{array}$ & $\begin{array}{c}43 \\
(62.32)\end{array}$ & 0.467 & $\begin{array}{c}38 \\
(55.07)\end{array}$ & $\begin{array}{c}27 \\
(39.13)\end{array}$ & 0.226 & $\begin{array}{c}27 \\
(39.13)\end{array}$ & $\begin{array}{c}38 \\
(55.07)\end{array}$ & 0.995 & $\begin{array}{c}26 \\
(37.68)\end{array}$ & $\begin{array}{c}38 \\
(55.07)\end{array}$ & 0.951 \\
\hline & Sim & $\begin{array}{c}5 \\
(29.41)\end{array}$ & $\begin{array}{c}12 \\
(70.59)\end{array}$ & & $\begin{array}{c}4 \\
(23.53)\end{array}$ & $\begin{array}{c}11 \\
(64.71)\end{array}$ & & $\begin{array}{c}7 \\
(41.18)\end{array}$ & $\begin{array}{c}9 \\
(52.94)\end{array}$ & & $\begin{array}{c}8 \\
(47.06)\end{array}$ & $\begin{array}{c}8 \\
(47.06)\end{array}$ & \\
\hline \multirow{2}{*}{$\begin{array}{l}\text { Beber água } \\
\text { enquanto } \\
\text { fala }\end{array}$} & Não & $\begin{array}{c}11 \\
(52.38)\end{array}$ & $\begin{array}{c}10 \\
(47.62)\end{array}$ & 0.139 & $\begin{array}{c}15 \\
(71.43)\end{array}$ & $\begin{array}{c}5 \\
(23.81)\end{array}$ & 0.155 & $\begin{array}{c}12 \\
(57.14)\end{array}$ & $\begin{array}{c}7 \\
(33.33)\end{array}$ & 0.223 & $\begin{array}{c}10 \\
(47.62)\end{array}$ & $\begin{array}{c}9 \\
(42.86)\end{array}$ & 0.724 \\
\hline & Sim & $\begin{array}{c}20 \\
(30.30)\end{array}$ & $\begin{array}{c}46 \\
(69.70)\end{array}$ & & $\begin{array}{c}28 \\
(42.42)\end{array}$ & $\begin{array}{c}33 \\
(50.00)\end{array}$ & & $\begin{array}{c}23 \\
(34.85)\end{array}$ & $\begin{array}{c}40 \\
(60.61)\end{array}$ & & $\begin{array}{c}25 \\
(37.88)\end{array}$ & $\begin{array}{c}37 \\
(56.06)\end{array}$ & \\
\hline
\end{tabular}

Teste de $\mathrm{Chi}^{2}$

*Significante

Também, houve significância estatística na correlação feita entre idade (mais velhos) e a queixa de garganta seca. Autores como Brunetti e Montenegro (2002) ${ }^{19}$ mencionaram em seus trabaIhos, que a hipossalivação está relacionada com a idade, uma vez que com o envelhecimento biológico, observa-se uma clara perda linear de células acinares, responsáveis pela produção de saliva, que são substituídas por gordura ou tecido conjuntivo. Portanto, a queixa de garganta seca pode ser justificada devido à xerostomia presente com o passar dos anos. Mais uma vez, destaque deve ser dado ao fato do intenso uso vocal demandado pelos docentes.

Ainda, em relação às alterações vocais correlacionadas a idade, de acordo com Boone $(1992)^{8}$, é 
mais comum ocorrer em sujeitos com idade mais avançada, uma vez que o período de máxima eficiência vocal acontece entre 25 e 45 anos $^{20}$. A partir desta idade uma série de alterações estruturais na laringe, com maior ou menor impacto, pode ser identificada, principalmente em relação à idade nas mulheres, pois, a partir dos 35 anos a mulher dá início a entrada no período da menopausa, momento em que as mudanças hormonais podem também interferir na voz ${ }^{20}$.

Nesta pesquisa não foi observada significância em nenhuma das autorreferencias a alteração vocal e sintomas, quanto ao tempo de magistério, número de escolas que trabalha, e a carga horária semanal de trabalho. Na literatura, há alguns estudos que evidenciam maior carga horária semanal ${ }^{21}$, e maior tempo de magistério ${ }^{10}$ como fatores que predispõem a alteração vocal.

Em relação aos fatores relativos ao estilo de vida (hábitos), embora a literatura afirme que tabagismo e etilismo são fatores que predispõem as alterações vocais, não foi encontrada associação estatisticamente significante neste estudo. Do mesmo modo, outros autores Ortiz et al (2004) $)^{22}$ também não observaram associação entre tabagismo e grau de disfonia, fato que parece predizer que esta questão não está presente entre os professores como um hábito que possa interferir nas questões da produção vocal. A porcentagem de professores que disseram fumar no presente $(6,82 \%)$ é mínima quando comparada à média de fumantes no país, a saber, $32,5 \%$ da população brasileira adulta ${ }^{23}$ Quanto aos hábitos relacionados a comportamentos vocais, foi possível observar significância na correlação entre autorreferência à alteração vocal e falar muito. $\mathrm{Na}$ direção contraria da pesquisa realizada por Ferreira et al $(2010)^{14}$, não foi possível observar associação estatisticamente significante na correlação entre problemas vocais e sintomas vocais com o número de refeições diárias, números de horas de sono, poupar a voz e gritar durante o uso profissional.

A ausência de associações pode ser explicada por vários aspectos: a amostra pesquisada não ser em numero representativo da população de professores do município de Sorocaba; as condições de trabalho num município do interior de São Paulo são diferentes das grandes capitais; e a necessidade de pesquisar outros fatores (ambientais e de organização de trabalho, por exemplo). Isso porque a porcentagem de sintomas vocais autorreferidos evidencia que esses professores desconhecem como a voz é produzida e os fatores que interferem nessa produção.

Mais uma vez fica registrada a necessidade do fonoaudiólogo criar condições de chegar até o professor para reverter seu quadro de adoecimento. A voz do professor é, sem dúvida, muito importante na mediação entre seus alunos, porém, sua formação não contempla informações e vivências que o auxiliem a estar atento a sua voz, com o objetivo de utilizá-la como um recurso importante na relação com seus alunos.

\section{CONCLUSÃO}

Dentre os fatores que podem predispor a alteração vocal, os entrevistados referiram em maior numero os hábitos de falar muito, em lugar aberto e gritar, e foi possível observar a associação entre a autorreferência a presença de alteração vocal e ser mulher e falar muito; entre cansaço ao falar e ser mulher e mais idoso; e entre garganta seca e ser mais idoso. 


\section{ABSTRACT}

Purpose: to identify the self-reported vocal habits of elementary and high-school teachers, and their correlation to the self-reported presence of hoarseness, strain when speaking, dry throat and presence of vocal disorders. Methods: 88 teachers from two public elementary and high schools of the city of Sorocaba answered the CPV-P questionnaire (Ferreira et al. 2007), made up of 84 questions. Data selected from this document to be used in this study were: functional situation, vocal aspects and lifestyle (habits) factors. For the statistical analysis the dependant variables were: reports of voice disorders, hoarseness, strain when speaking and dry throat. The independent variables were gender, age, functional situation, and habits related to vocal use. The statistical analysis was performed using STATA 8.0 software, employing the association test (chi-squared) with $p \leq 0.05$. Results: $64.77 \%$ of the subjects self-reported vocal disorder, $54.55 \%$ strain when speaking, $53.41 \%$ dry throat, and $44.32 \%$ reported hoarseness. As far as vocal habits, $86.52 \%$ reported speaking excessively, $59.55 \%$ speak in open spaces, $50.56 \%$ reported yelling, $28.41 \%$ smoking, $28.09 \%$ speak while carrying heavy loads and $22.73 \%$ reported ingestion of alcohol. However, $67.42 \%$ report sparing their voices and $75.28 \%$ ingest water during vocal use. Conclusion: the most frequently self-reported habits were excessive speaking, speaking in open spaces and yelling. Among the variables, it was possible to observe an association between self-reported vocal disorder and being a woman and excessive speaking; between strain when speaking and being a woman of older age; and between dry throat and older age.

KEYWORDS: Faculty; Voice Disorders; Risk Factors

\section{REFERÊNCIAS}

1. Dragone MLS, Behlau M. A fonoaudiologia brasileira e a voz do professor - olhares científicos no decorrer do tempo. Fonoaudiol Brasil. 2006;4(2):6-9.

2. Araújo TM, Reis EJFB, Carvalho FM, Porto LA, Reis IC, Andrade JM. Fatores associados a alterações vocais em professoras. Cad. Saúde Pública. 2008; 24(6): 1229-38.

3. Roy N, Merrill RM, Gray SD, Smith EM. Voice disorders in the general population: prevalence, risk factors, and occupational impact. Laryngoscope. 2005; 115(11):1988-95.

4. Jardim R, Barreto SM, Assunção AA. Voice Disorder: case definition and prevalence in teachers. Rev Bras Epidemiol. 2007; 10(4):625-36.

5. Romano CC, Alves LA, Silva LA da, Marziale MHP, Robazzi MLCC. Alterações vocais decorrentes do trabalho em professores: uma revisão de literatura. Rev Enferm UFPE On Line. 2009 jul./set.;3(3):69-277.

6. Sliwinska-Kowalska M, Niebudek-Bogusz E, Fiszer M, Los-Spychalska T, Kotylo P, SznurowskaPrzygocka $\mathrm{B}$ et al. The prevalence and risk factors for occupational voice disorders in teachers. Folia Phoniatr Logop. 2006; 58(2):85-101.

7. Medeiros AM, Barreto SM, Assunção AA.. Voice disorder (dysphonia) in public school female teachers working in Belo Horizonte: prevalence and associated factors. J Voice. 2008; 22(6):676-87.

8. Boone DR. Inimigos biológicos da $\mathrm{Voz}$ Profissional. Pró-Fono. 1992; 4(2):3-8.

9. Ferreira LP. Assessoria Fonoaudiológica aos Profissionas da Voz. In: Ferreira LP, Lopes DMB, Limongi SCO, organizadores. Tratado de fonoaudiologia. 2. ed. São Paulo: ROCA. 2009. p. 138-49.

10. Grillo MHMM, Penteado RZ. Impacto da voz na qualidade de vida de professore(a)s do ensino fundamental (original title: The impact of voice on the quality of life of elementary school teachers). Pró-Fono. 2005 set-dez; 17(3):321-30.

11. Carneiro SAM. Saúde do trabalhador público: questão para a gestão de pessoas - a experiência na Prefeitura de São Paulo. Revista do Serviço Público. 2006; 57(1): 23-49.

12. Ferreira LP, Giannini SPP, Latorre MRDO, Zenari MS. Distúrbio de voz relacionado ao trabalho: proposta de um instrumento para avaliação de professores. Rev. Dist Comunicação. 2007; 19(1): 127-36.

13. Alves LA, Robazzi MLCC, Marziale MHP, Felippe ACN, Romano CC. Alterações da saúde e da voz do professor, uma questão de saúde do trabalhador. Rev Latina - Am. Enfermagem. 2009 julho-agosto; 17(4): 566-72. 
14. Ferreira LP, Latorre MRDO, Giannini SPP, Ghirardi ACAM, Karmann DF, Silva EE. Influence of abusive vocal habits, hydration, mastication, and sleep in the occurrence of vocal symptoms in teachers. Journal of Voice. 2010; 24(1): 86-92.

15. Ferreira LP, Beneditti PH. Condições de produção vocal de professores de deficientes auditivos. Rev. CEFAC. 2007; 9(1):79-89.

16. Tavares ELM, Martins RHG. Vocal evaluation in teachers with or whithout symptoms. Journal of Voice. 2007; 21(4): 407-14.

17. Ortiz E, Costa EA, Spina AL, Crespo NA. Proposta de modelo de atendimento multidisciplinar para disfonias relacionadas ao trabalho: estudo preliminar. Rev Bras Otorrinolaringol. 2004; 70(5):590-6.

18. Soares EB, Borba DT, Barbosa TK, Medved DM, Montenegro ACA. Hábitos vocais em 2 grupos de idosos. Rev. CEFAC. 2007; 9(2):221-7.
19. Brunetti RF, Montenegro FLB. Odontogeriatria: noções de interesse clínico. São Paulo: Artes Médicas. 2002.

20. Behlau M, Azevedo R, Pontes P. Conceito de Voz normal e classificação das disfonias. In: Behlau M. Voz: o livro do especialista. Rio de Janeiro: Revinter; 2001. p.53-84.

21. Penteado RZ, Bicudo-Pereira IMT. Qualidade de vida e saúde vocal de professores. Rev Saúde Pública. 2007; 41(2):236-43.

22. Ortiz E, Lima EA, Costa EA. Saúde Vocal de Professores da Rede Municipal de Ensino de Cidade do Interior de São Paulo. Rev. Bras. Med. Trab. 2004; 2(4):263-6.

23. Gigliotti A, Laranjeira R. Hábitos, atitudes e crenças de fumantes em quatro capitais brasileiras. Rev. Bras. Psiquiatr. 2005; 27(1):37-44.

DOI: 10.1590/S1516-18462010005000099

RECEBIDO EM: 20/01/2010

ACEITO EM: 28/06/2010

Endereço para correspondência:

Carollina Caporossi

Rua Padre João Manoel, 202, Apt. 125,

Edifício Saint German

São Paulo - SP

CEP: 01411-000

E-mail: carollinacaporossi@gmail.com 